\title{
Study Pulse Parameters versus Cavity Length for Both Dispersion Regimes in FM Mode Locked
}

\author{
Bushra R.Mhdi*, Gaillan H.Abdullah, Nahla A.Aljabar, Basher R.Mhdi \\ Ministry of Science and Technology, Iraq, Bagdad \\ e-mail: boshera65m@yahoo.com
}

\begin{abstract}
To demonstrate the effect of changing cavity length for FM mode locked on pulse parameters and make comparison for both dispersion regime, a plot for each pulse parameter as Lr function are presented for normal and anomalous dispersion regimes. The analysis is based on the theoretical study and the results of numerical simulation using MATLAB. The effect of both normal and anomalous dispersion regimes on output pulses is investigat Fiber length effects on pulse parameters are investigated by driving the modulator into different values. A numerical solution for model equations using fourth-fifth order, Runge-Kutta method is performed through MATLAB 7.0 program. Fiber length effect on pulse parameters is investigated by driving the modulator into different values of lengths. Result shows that, the output pulse width from the FM mode locked equals to $t=501 \mathrm{~ns}$ anomalous regime and $t=518 \mathrm{~ns}$ in normal regime.
\end{abstract}

Keywords: FM mode lock, cavity length, dispersion regimes

\section{Introduction}

Ultrashort optical pulses have great applications in fields such as ultrafast optics, optical fibre communication, optical measurement, micro-mechanism processing, and medical treatments [1]. Compared with mode-locked solid-state lasers, ultrashort pulse fibre lasers have advantages of compactness, greater stability and so on. So far, researchers have proposed soliton fibre lasers, [2] stretched pulse fibre lasers [3] and self similar fibre laser. [4-5] But the evolution mechanisms of these pulse fibre lasers fail in output pulse energy or complexness (as dispersion compensation components, such as prism pairs, grating pairs, chirp mirrors, and micro construction fibres, are used). Recently, a novel all-normal-dispersion (ANDi) modelocked fibre laser, in which all components are of normal dispersion, has been proposed [6]. In this diagram, the energy of output pulses can be much higher, [7-10] and designs can be greatly simplified especially for Yb-doped mode-locked fibre lasers whose operating wavelength is in the normal dispersion domain of common fibers. Ultrashort fiber lasers offer additional benefits such as small physical dimensions, increased stability under environmental conditions, reduced thermal management and diffraction limited beam quality. In addition, fiber lasers can be easily engineered and are usually less expensive than their solid-state lasers counterparts. Particularly, Yb3+ doped fibers have a broad emission bandwidth, large saturation fluence and high optical to optical conversion efficiency. Therefore, they form an excellent gain medium for the generation and amplification of ultrashort optical pulses in the $1 \mu \mathrm{m}$ wavelength range [11].

\section{Theoretical Concept}

Depending on master equation Eq.(1), and using the assumed pulse shapes for both dispersion regimes after modifying Ginzburg-Landau Equation, GLE, by adding TOD and mode-locker effects, the extended solution will be as in the following relations [12-15].

$$
T_{R} \frac{\partial A}{\partial T}+\frac{1}{2}\left(\overline{\beta_{2}}+i \bar{g} T_{2}^{2}\right) L_{R} \frac{\partial^{2} A}{\partial t^{2}}-\frac{\overline{\beta_{3}}}{6} L \frac{\partial^{3} A}{\partial t^{3}}=\overline{\gamma L}|A|^{2} A+\frac{1}{2}(\bar{g}-\bar{\alpha}) L A+M(A, t)
$$

In the following all the terms of Mode-Locking master equation will be identified. 


\subsection{Normal Regime}

$$
A(T, t)=a\left(\exp \left(-\frac{(\Phi-\xi)^{2}}{2 \tau^{2}}\right)^{1+i q} \times \exp \left(i \Omega(t-\xi)+i K T+i \varphi_{Q}\right.\right.
$$

\subsection{Anomalous Regime}

$$
A(T, t)=a\left(\operatorname{sech}\left(\frac{t-\xi}{\tau}\right)\right)^{1+i q} \times \exp \left(i \Omega(t-\xi)+i K T+i \varphi_{Q}\right.
$$

Where pulse parameters for both profiles are:
(a) represents pulse amplitude,
(६) Temporal shift,
$(\tau)$ pulse width,
$(q)$ Chirp,
$(\Omega)$ frequency shift,
$i K T+i \varphi_{Q}$ Represent the phase and rarely is of physical interest in lasers producing picoseconds pulses, and will be ignored [12].
The relations of pulse parameters with temporal pulse profile are as following $[16,17]$ :

$$
\begin{aligned}
& E(T)=\int_{-\infty}^{+\infty}|A(T, t)|^{2} d t \\
& \xi(T)=\frac{1}{E} \int_{-\infty}^{+\infty} t|A(T, t)|^{2} d t \\
& \Omega(T)=\frac{t}{2 E} \int_{-\infty}^{+\infty}\left[A \frac{d A}{d t}-A \frac{d A}{d t}\right]^{2} d t \\
& q(T)=\frac{t}{E} \int_{-\infty}^{+\infty}(t-\xi)\left[A \frac{d A}{d t}-A \frac{d A}{d t}\right]^{2} d t \\
& (T)^{2}=\frac{2}{E} \int_{-\infty}^{+\infty}(t-\xi) \mid A(T, t)^{2} d t
\end{aligned}
$$

\section{Results and Analysis}
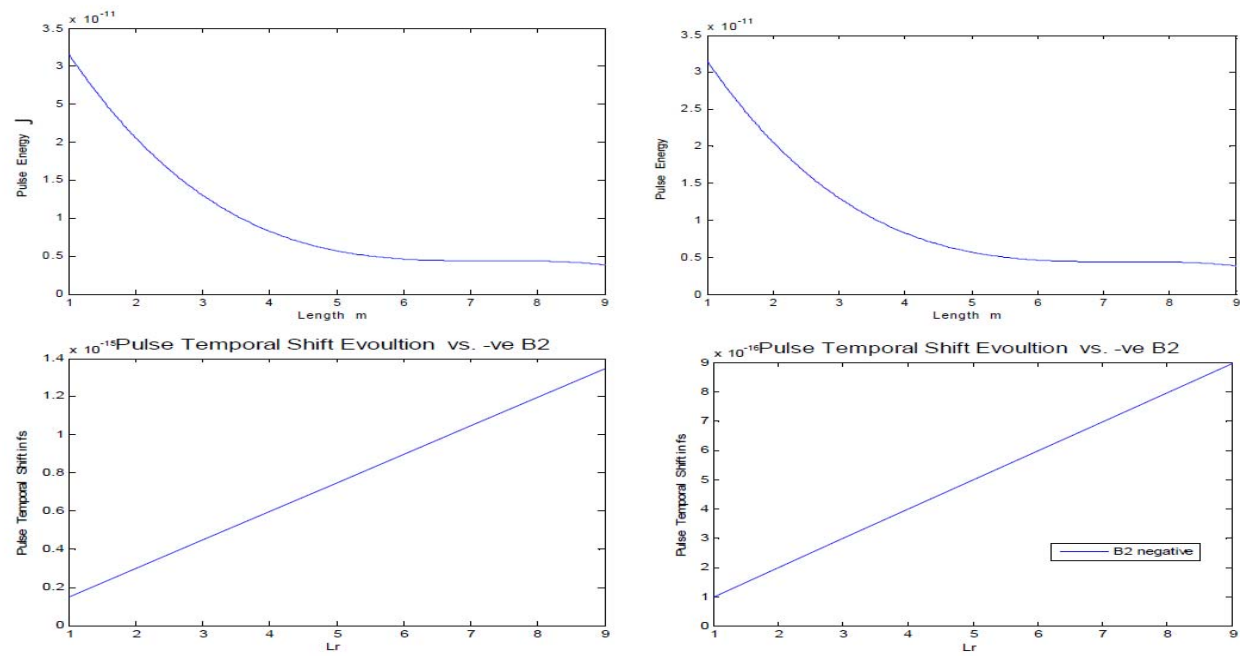

(a)

(b)

Figure 1. Pulse energy, temporal shift versus cavity length in (a) Normal, and (b) Anomalous dispersion 

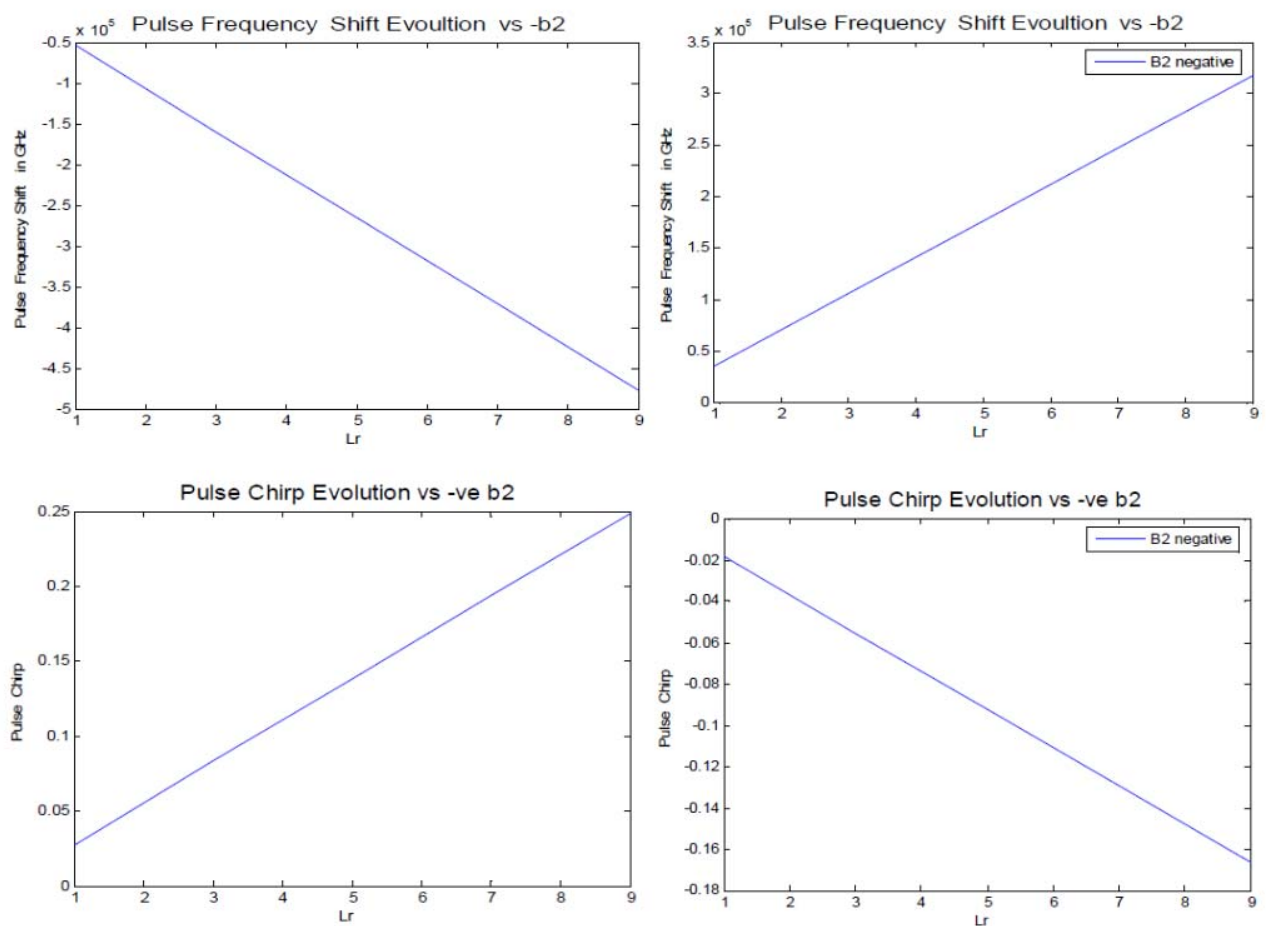

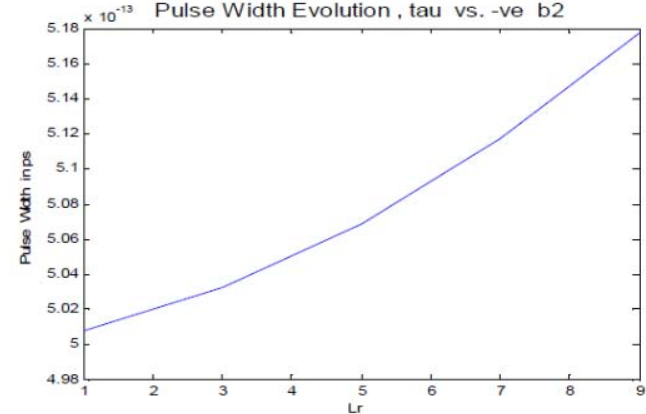

(a)

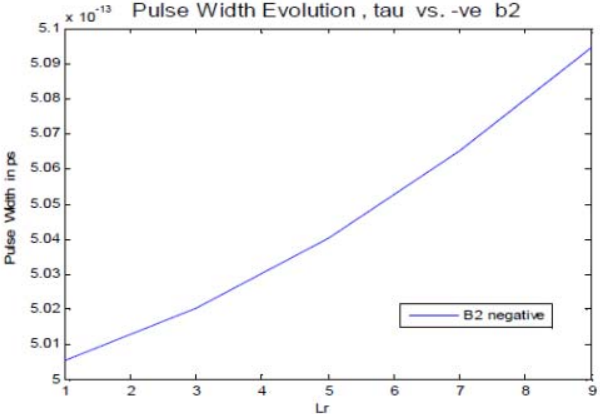

(b)

Figure 2. Frequency shift, chirp and width versus cavity length in (a) Normal, and(b) Anomalous dispersion

To demonstrate the effect of changing cavity length on pulse parameters and make comparison for both dispersion regimes, a plot for each pulse parameter as Lr function is drawn in Figure 1 for normal and anomalous dispersion regimes. As shown in Figure 1 almost same behavior for pulse energy is seen for variable Lr. Energy decreases exponentially as $L r$ increases. Then decreasing rate after certain value of cavity length $(\mathrm{Lr}=5 \mathrm{~m})$ and becomes so small for high values and almost constant straight line. Temporal shift plots in Figure 1 for both regimes are shown almost the same behavior in both regimes. Pulse temporal shift is increases linearly as $L r$ increases. The main difference is that: in anomalous regime, the temporal shift is greater than normal regime by five times. Inspecting plots for pulse frequency shift in Figure 2 the linear relation in anomalous and normal regime is seen. For normal regime, the frequency shift decrease linearly with $\mathrm{Lr}$ increases, while in anomalous regime, frequency shift increases with increase Lr.

For pulse chirp as function of cavity length, as shown in Figure 2 the pulse chirp in normal regime suffers from positive linear relation for variable $L r$ increasing with increasing of $L r$ .In anomalous regime, the negative linear relation chirp decreases with increasing Lr. This linear chirp enables us to compare the pulse outside the laser cavity without deforms in an anomalous dispersion regime until free chirp pulse is introduce [12]. Cavity Length has great effect on pulse

IJEEI Vol. 3, No. 1, March 2015: $30-33$ 
width since it is responsible for mode-locking mechanism and producing pulses train. As shown in Figure 2, pulse width increases exponentially with the increase of $\mathrm{Lr}$, but for anomalous regime it is much faster increasing in magnitude and less in $L r$ values as compared with normal regime. Almost, for anomalous regime, the minimum pulse width is obtained.

\section{Conclusion}

The length cavity varying of FM mode locking pulse parameter effect directly with varying $L r$. The effective length in FM is equal $4 \mathrm{~m}$. Pulse width increases exponentially with the increase of $L r$, but for anomalous regime it is much faster increasing in magnitude and less in $L r$ values as compared with normal regime. Almost, for anomalous regime, the minimum pulse width is obtained.

\section{References}

[1] U Keller, et al. Self-starting and reliable modelocking. Opt. Lett. 1992; 17: 505.

[2] V Matsas, T Newson, D Richardson, D Payne. vibrating soliton pairs in a mode- locked laser cavity. Optics Letters. 2006; 31(14): 2115-2117.

[3] H Haus, K Tamura, L Nelson, E Ippen.All normal - dispersion Yb-doped mode-locked fiber. IEEE J. Quantum Electron. 1995; 31: 591.

[4] FÖ Ilday, JR Buckley, WG Clark, FW Wise. All- fiber- integrated soliton- similiration laser with in line fiber filter. Phys. Rev. Lett. 2004; 92: 213902.

[5] Y Deng, Tu CH, Lu FY. All-normal dispersion Yb-doped mode-locked fiber. Acta Phys Sin. 2009; 58 : 3173.

[6] A Chong, J Buckley, W Renninger, FW Wise. All-normal- dispersion femtosecond fiber laser. Opt. Express. 2006; 14: 10095.

[7] A Chong, W Renninger, F Wise. All-normal-dispersion femtosecond fiber laser with pulse energy above 20 nJ. Opt. Lett. 2007; 32: 2048.

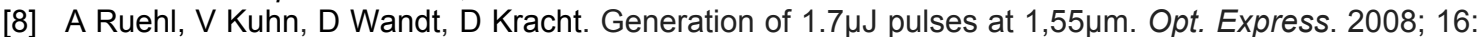
3130.

[9] BW Liu, Hu ML, YJ Song, L Chai, QY Wang. Approaching 100nJ pulse energy output from a modelocked. Acta Phys. Sin. 2008; 57: 6921.

[10] Y J Song, Hu M L, Q W Liu, J Y Li, W Chen, L Chai and Q Y Wang, "Dispersion- soliton mode-locked laser based on large-mode-area" Acta Phys. Sin. 57 , (2008).5045.

[11] A Chong, W Renninger, F Wise. Properties of normal-dispersion femtosecond fiber lasers. Opt. Soc. Am. 2008.

[12] G Nicholas Usechak, P Govind Agrawal, D Jonathan Zuegel. FM Mode-Locked Fiber Lasers Operating in the Autosoliton Regime. IEEE Journal of Quantum Electronics. 2005; 41(6).

[13] Xiangyu Zhou, Dai Yoshitomi, Yohei Kobayashi, Kenji Torizuka. Generation of 28-fs pulses from a mode-locked ytterbium fiber oscillator. Optics Express. 2008; 16(10): 2545-2505.

[14] G Nick Usechak, P Govind Agrawal, D Jonathan. Zuegel. Tunable high-repetition-rate, harmonically mode-locked ytterbium fiber laser. Optics Letters. 2004; 29(12): 2350-2365.

[15] J Jennifer ONeil. Pulse Dynamics in Actively Modelocking Fiber Optic Lasers. Honors Thesis Spring. University of Washington, Applied and Computational Mathematical Sciences. 2002.

[16] G Nicholas Usechak, P Govind Agrawal. Rate-equation approach for frequency-modulation mode locking using the moment method. Opt. Soc. 2005; 22(12).

[17] G Nicholas Usechak, P Govind Agrawal. Semi-analytic technique for analyzing mode-locked lasers. Optics Express. 2005; 13(6): 2075-2081. 